\title{
Quaderni
}

QUADERN I Communication, technologies, pouvoir

70 | Automne 2009

Think tanks, experts et pouvoirs

\section{La chevalerie, un mythe à l'ère de la communication}

Céline Bryon

\section{CpenEdition}

Édition électronique

URL : http://journals.openedition.org/quaderni/520

DOI : 10.4000/quaderni.520

ISSN : 2105-2956

Éditeur

Les éditions de la Maison des sciences de l'Homme

Édition imprimée

Date de publication : 5 octobre 2009

Pagination : 111-119

Référence électronique

Céline Bryon, «La chevalerie, un mythe à l'ère de la communication », Quaderni [En ligne], 70 | Automne 2009, mis en ligne le 05 octobre 2011, consulté le 19 avril 2019. URL : http://journals.openedition.org/ quaderni/520; DOI : 10.4000/quaderni.520

Tous droits réservés 


\section{Communication}

\section{la chevalerie, communication}

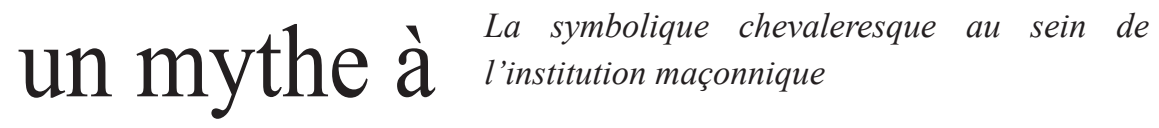
l'ère de la Execrant une profonde fassination sur les

Maître de conférences Institut National Polytechnique de Toulouse esprits, la chevalerie médiévale a donné naissance à un mythe qui ponctue l'histoire de

\section{Les multiples résurrections d'un mythe}

l'Occident et réapparaît de manière sporadique, sous différentes formes et à diverses occasions. Après la disparition de cet ordre, qui déclina dès la fin du Moyen Âge, la littérature a continué de véhiculer le système de valeurs traditionnelles qui lui est attaché : courage, piété, sens de l'honneur et du devoir, noblesse des sentiments, esprit de sacrifice et loyauté... À cet égard, Don Quichotte, le personnage anachronique de Cervantès, est tout à fait significatif. Il inaugure une longue série de figures, fictives ou réelles, nostalgiques du temps béni où les chevaliers défendaient la veuve et l'orphelin, perchés sur leur fier destrier, se battaient en duel pour l'amour de quelque gente dame et combattaient pour délivrer la Terre sainte des mains des infidèles. Ainsi le duc de Richelieu proclamaitil: «Heureux temps chevaleresques, époque à jamais mémorable, soyez présents à notre mémoire et la fausse grandeur du XVIII e siècle s'éclipsera devant votre véritable gloire $[\ldots]$ Siècles chevaleresques, dans votre heureuse innocence, la religion, l'honneur et la vertu furent les divins flambeaux qui vous éclairèrent et servirent à vous guider ». Dans le cadre des guerres de conquête de la Grande armée, Stendhal déclare à son tour dans La Chartreuse de Parme: «Il défaisait un à un tous ses beaux rêves d'amitié chevaleresque et sublime, comme celle des héros de la Jérusalem libérée. [...] La 
guerre n'était donc plus ce noble et commun élan d'âmes amantes de la gloire qu'il s'était figuré d'après les proclamations de Napoléon!».

Ces idéaux, nombre d'institutions - au rang desquelles on compte la franc-maçonnerie-, s'efforcèrent de les ressusciter. Lors de leur apparition au début du XVIII ${ }^{\mathrm{e}}$ siècle, en effet, les premières loges assirent une large part de leur symbolisme sur l'ancienne chevalerie. Elles firent de cette dernière l'un de leurs mythes fondateurs, lui empruntant certaines de ses armes (l'épée devint un objet du rituel) et de ses pratiques initiatiques (le profane et futur adepte se voit sacré franc-maçon par une cérémonie solennelle proche de l'adoubement, après avoir subi une série d'épreuves). Ses hauts grades mêmes portent la marque de cette influence : le grade de Chevalier de l'Orient, ou Chevalier de l'Epée, est attesté en France dès 1748. Quant au $30^{\mathrm{e}}$ degré du Rite écossais ancien et accepté, dit grade du Chevalier Kadosh, apparu en 1749, il renoue avec la légende templière. Au-delà des symboles, les loges voyaient probablement dans la loyauté et la solidarité qui animaient les membres de la communauté chevaleresque (cette fraternitas militaire qui caractérisait le comitatus), une parenté avec leurs propres aspirations fraternalistes.

En se créant une telle filiation, en ancrant sa démarche spéculative dans une époque reculée, auréolée de gloire pour ses faits d'armes exemplaires, la franc-maçonnerie insufflait à ses activités un caractère concret, presque opératif ${ }^{1}$. Elle se donnait une légitimité qui fait souvent défaut aux institutions naissantes, ainsi que le montre Eric Hobsbawm dans un ouvrage intitulé
L'Invention de la tradition. Mais surtout, elle s'entourait d'une importante charge imaginaire. Comme le remarque Pierre Mollier, le fait que le mythe de la chevalerie ait pu resurgir au siècle de Voltaire, au sein d'une structure qui s'efforce de réhabiliter les formes symboliques et le besoin d'onirisme inhérent à la condition humaine, ne saurait être un hasard. La chevalerie, en effet, représente des valeurs en voie de désaffection. Elle offrit à la franc-maçonnerie « un cadre et un support à ces «connaissances précieuses et secrètes» qui-paradoxe du siècle-fascine l'homme des Lumières, critique vis-à-vis des institutions religieuses, mais frustré par l'émergence du rationalisme moderne $»^{2}$.

La résurrection du mythe chevaleresque opérée par la franc-maçonnerie est, à bien des égards, annonciatrice du mouvement romantique, lequel poursuivit cette entreprise de réhabilitation. Walter Scott, lord Byron et Alexandre Dumas, pour ne citer que les auteurs les plus célèbres, participeront de la diffusion du mythe, alors même que la révolution industrielle, avec ses premiers chemins de fer et ses armes de plus en plus sophistiquées, paraît frapper d'obsolescence l'image du guerrier médiéval, qui combat à l'arme blanche sur son cheval. Sous l'Empire, Napoléon Bonaparte, lui aussi, introduisit des titres honorifiques dans lesquels on recense le grade de Chevalier, et ce afin de reconstituer une élite - substitut de l'ancienne noblesse - que la chute de l'Ancien Régime avait largement décimée; mais également afin de consolider son propre pouvoir par de tels actes de reconnaissance symboliques ${ }^{3}$. L'ordre de la Légion d'honneur en est une illustration. Aujourd'hui encore, ce grade subsiste au sein 
des ordres nationaux et des ordres de mérite ministériels, constitués par l'ordre des Palmes académiques, l'ordre des Arts et des Lettres, l'ordre du Mérite agricole et l'ordre du Mérite Maritime.

Les chevaliers de l'air, mythe fondateur de l'aviation militaire et substrat de propagande politique

Le mythe fit une nouvelle percée à partir de la Première Guerre mondiale, au sein de l'aviation de chasse. À terre, les exploits individuels et la «belle mort», qui prévalaient dans les récits épiques puis les chansons de geste, ne sont plus permis aux soldats ${ }^{4}$. Les poilus meurent anonymement dans la boue des tranchées, fauchés par des projectiles frappant au hasard, ainsi que le souligne Jean-Pierre Dournel ${ }^{5}$. La littérature dénonce l'horreur de la guerre technologique, à travers les œuvres de Henri Barbusse ( $\mathrm{Le} \mathrm{Feu}$ ) ou de Louis-Ferdinand Celine (Voyage au bout de la nuit). Aux antipodes, les aviateurs dominent le monde et se battent en combat singulier, renouant avec les traditions guerrières malgré la modernité des équipements, représentant une guerre prétendue propre dans la sale guerre. La presse, puis la population, vantent alors les mérites de ceux que l'on baptise "les Chevaliers de l'air», narrant les prouesses des as de la Grande Guerre et autres pionniers de l'aéronautique: Georges Guynemer, Roland Garros, Jean Mermoz, René Fonck, Dorme, Nungesser... L'adulation du public est telle qu'aux héros de l'Histoire, bientôt transformés en héros de légende par une poignée de biographes-hagiographes, vont s'ajouter des héros de fiction. Ainsi en est-il de
Tanguy et Laverdure, personnages de B.D avant que de devenir les protagonistes d'un feuilleton radiophonique, puis télévisé, à l'origine de bien des vocations; puis de Top Gun, production cinématographique qui perpétua le mythe tout en l'actualisant, l'avion à réaction remplaçant alors l'avion à hélice, comme le montra Roland Barthes dans un chapitre intitulé «L'HommeJet ».

Georges Guynemer, l'as aux 53 victoires, incarna longtemps à lui seul le mythe du chevalier de l'air. Le journaliste Jacques Mortane, mais surtout l'écrivain Henry Bordeaux, avec son œuvre Vie héroïque de Guynemer : le chevalier de l'air, contribuèrent à son aura. Désireux d'insuffler une connotation épique au destin du pilote, l'auteur découpe sa biographie en chants. Et de déclarer: "L'enfance batailleuse d'un Guynemer rejoint celle d'un Roland, d'un Duguesclin, d'un Bayard». Le général Paul Anthoine, quant à lui, le désigne comme «un chevalier de l'air sans peur et sans reproche». Guynemer joue avant tout un rôle de référent. Pour une aviation militaire encore balbutiante, mais qui commence à laisser entrevoir tous les avantages que l'armée peut tirer de la supériorité aérienne (que l'on confinait d'abord à des missions d'observation, de surveillance et de reconnaissance, au même titre que les ballons dirigeables), rapidement déclinée en outil de liaison avec les troupes et surtout en arme offensive dédiée au bombardement et au combat, le jeune capitaine offre un visage concret au mythe - encore un peu abstrait - des chevaliers de l'air. Cette mythification s'avérait nécessaire non seulement à cause de la jeunesse de l'aviation, mais également de son aspect 
technologique, peu propice à la création d'un quelconque lien social ${ }^{6}$. En s'appuyant sur une tradition incontestée, l'institution se fabriqua une continuité dans le temps et une cohésion infra-groupale. François Pernot souligne ainsi : "Guynemer-chevalier du ciel devient donc un authentique archétype, un portrait de référence, un véritable cas expérimental de reprise et de diffusion du modèle chevaleresque comme creuset identitaire $»^{7}$.

Une nouvelle communauté guerrière s'établit, en effet, qui a besoin de ses propres symboles et rites. Il s'agit d'un mythe fondateur, attractif et mobilisateur, comme ce fut le cas pour les premières loges maçonniques. Encore une fois, la filiation symbolique s'accompagna d'une filiation historique, aux fondements parfois plus imaginaires que réels. Les aviateurs euxmêmes s'approprièrent ces prétendues origines chevaleresques d'autant plus naturellement qu'ils furent rattachés à l'armée de terre et entretinrent des liens étroits avec la cavalerie ${ }^{8}$, avant d'être intégrés à une entité autonome, l'armée de l'air, à partir de 1933. En outre, la notion de caste qui leur est inhérente n'était pas pour leur déplaire, car elle leur conférait un caractère d'exceptionnalisme. Il est vrai qu'on voue un véritable culte à ces chevaliers de l'air, tant au niveau de la communauté militaire que de la nation. Georges Guynemer est un héros national, admiré par les jeunesses françaises de l'entre-deux-guerres. En 1922, une inscription en son nom est inaugurée au Panthéon par Raymond Poincaré. Un timbre poste paraît à son effigie en 1940. Le régime de Vichy se sert même du souvenir toujours vivace du défunt pilote pour en faire un modèle de courage et de perfection morale à imiter, à travers la presse qui reçoit des directives strictes du ministère d'État à la Défense nationale ${ }^{9}$. Un système de communication, voire de propagande, se met en place autour du mythe des chevaliers de l'air, sachant que le mythe est déjà «un système de communication » à part entière, porteur d'« un message », ainsi que l'a montré Roland Barthes.

Quels sont précisément les messages que diffuse ce mythe? En interne, auprès du personnel navigant mais également de l'ensemble des forces armées, il est un facteur de motivation, un catalyseur destiné à entretenir la vocation des militaires ainsi que leur capacité d'abnégation. La citation officielle de Georges Guynemer, gravée sur une stèle de l'école de l'air (chargée de former les futurs officiers de l'institution) et lue lors des prises d'armes annuelles en l'honneur du chasseur, est un panégyrique. Elle se présente aussi comme une incitation au dépassement de soi, affirmant que le défunt capitaine « exaltera l'esprit de sacrifice et provoquera les plus nobles émulations». En externe, il est un symbole d'héroïsme patriotique, un facteur de ralliement national. Ce n'est d'ailleurs pas en vain que l'on compare parfois Guynemerà Jeanne d'Arc, allant jusqu'à inventer au pilote une chasteté que son aventure avec la comédienne Yvonne Printemps dément, mais que la pureté attachée au mythe $\mathrm{du}$ chevalier semble exiger. Parallèlement aux schèmes inconscients présents dans la mémoire collective, s'impose une instrumentalisation politique, consciente et volontaire. Le 11 septembre 1941, Paris-Soir déclare que «le nom et la personne de Guynemer peuvent servir d'exemple en un temps où la France n'a pas 
trop de toutes ses gloires », et le 13 septembre 1941, Le Progrès de Lyon affirme : "C'est ainsi que Guynemer devrait être pour tous les jeune Français, quelles que soient leurs activités, un magnifique modèle d'acharnement dans l'effort. Il devrait être pour eux un saint que l'on invoque au réveil et dont la vie serait toujours présente à la pensée ${ }^{10}$.

\section{Les raisons d'un indéfectible succès}

Du mythe de l'âge d'or au mythe du sauveur...

Dans Les Origines de la pensée grecque, JeanPierre Vernant souligne la scission qui est intervenue, au sein de la culture hellenistique du $\mathrm{V}^{\mathrm{e}}$ siècle avant Jésus-Christ, au niveau des cadres de la pensée et du discours. Le muthos, définie comme fable, se trouve dès lors dévalorisé par rapport au logos, parole profane reposant sur une activité de la raison et une démarche démonstrative. Pourtant, ce discrédit jeté par la philosophie sur le champ de l'imaginaire, par opposition au champ scientifique, n'a guère empêché la société occidentale de ressusciter les mythes du passé, voire de construire de nouvelles formes de mythification, ainsi que l'a démontré Roland Barthes dans ses Mythologies. N'est-ce pas parce qu'il satisfait à un profond besoin de spiritualité, fonction que la science moderne ne parvient pas à remplir et à laquelle elle contrevient même dans une certaine mesure ? La figure du chevalier, qui possède une puissance évocatrice, s'inscrit dans cette veine. Elle relève de la catégorie des mythes de « l'âge d'or » qui exalte « la pureté des origines », telle qu'analysée par Raoul Girardet dans Mythes et mythologies politiques. À travers elle, le passé se trouve sublimé, synonyme de perfection par opposition à un présent décadent. Mieux encore, par l'héroïsme qui lui est inhérent, l'image du chevalier se révèle salvatrice. Par ses considérations sotériologiques, voire eschatologiques, elle rejoint un autre grand mythe également étudié par Raoul Girardet, à savoir le « mythe du sauveur ». Les chevaliers de l'air en témoignent, incarnations d'une celeritas qui rééquilibre la nostalgie des origines dont le mythe chevaleresque est porteur.

L'on ne s'étonnera donc pas de ce que la représentation magnifiée du chevalier réapparaisse à des moments particulièrement critiques (ce qui tend à accréditer la thèse soutenue par Raoul Girardet, à savoir que le mythe serait l'«expression d'un malaise social », ainsi qu'une entreprise simultanée visant à lutter contre ce mal) : le rationalisme des Lumières, privatif des repères religieux qui guidaient jusqu'alors le plus grand nombre; la Première Guerre mondiale, qui inaugure les combats technologiques et les massacres de masse ; le régime de Vichy, qui collabore avec l'Allemagne nazie, au mépris des idéaux de justice et de liberté que la Révolution française avait mit à l'honneur. Ces périodes disruptives de l'Histoire se caractérisent par une crise d'identité, que le mythe chevaleresque, facteur de restructuration mentale et de cohésion sociale, jugule. Comme le remarque Franco Cardini, «dans l'imaginaire de l'Occident, le chevalier est une figure intemporelle ${ }^{11}$, une sorte d'image archétypale, au sens jungien. Il apparaît donc naturel que le chevalier ait été mythifié, étant entendu que le mythe lui-même prétend à l'intemporalité et à l'universalité, 
raison pour laquelle les principes de répétition et de variation y sont prégnants, à travers ce que Gilbert Durand nomme des « mythèmes ». ${ }^{12}$

Cependant, il convient de préciser que la représentation que l'opinion publique possède de la chevalerie est quelque peu fallacieuse. La réalité était bien différente de ce que l'on s'est plu à imaginer et à diffuser au cours des siècles. Initialement, les chevaliers étaient des soudards et des pillards, qui considéraient la guerre comme un moyen de subsistance et un instrument de promotion sociale ${ }^{13}$. Il s'agissait souvent d'une forme de mercenariat, fort éloignée des actes désintéressés ou guidés par la foi que la mémoire collective véhicule. L'idéologie chevaleresque qui fait tant fantasmer, si elle possède quelque fondement historique indéniable, relève malgré tout davantage de la fiction. Ce n'est qu'à partir du XII ${ }^{\mathrm{e}}$, voire du XIII ${ }^{\mathrm{e}}$ siècle, que les milites commencèrent à se doter d'une éthique et à se muer en élite (et encore ne fut-ce que de manière progressive et inégale).

Une "politique de la mémoire " : l'introduction du sacré à l'époque du tout-technologique

La dimension sacrée, voire mystique, que l'on perçoit en filigrane dans la chevalerie, et dont le cycle arthurien est l'illustration manifeste, n'est sans doute pas étrangère à l'incroyable fortune de ce mythe. Tel est d'ailleurs le point de divergence majeur entre la chevalerie et la cavalerie. Quête d'absolu, tout du moins dans l'inconscient collectif qui réduit volontiers les activités chevaleresques à d'éternelles quêtes du Graal, le mythe était promis à une longue prospérité, d'autant plus forte que la société moderne se caractérise par un « désenchantement du monde », selon le mot de Max Weber. Des penseurs tels Ernst Cassirer, Gaston Bachelard ou encore Gilbert Durand, ont montré l'utilité des formes mythiques au sein d'une entreprise générale de démythification, où le concept évince le symbole. Paul Ricœur, mettant en évidence le rôle compensatoire qu'endosse de nos jours la réutilisation croissante des symboles, déclare ainsi : «si nous soulevons le problème du symbole maintenant, à cette période de l'histoire, c'est en liaison avec certains traits de notre "modernité» et pour riposter à cette modernité même. Le moment historique de la philosophie du symbole, c'est celui de l'oubli et aussi celui de la restauration: oubli des hiérophanies; oubli des signes du Sacré $\gg{ }^{14}$.

La résurrection de la chevalerie s'inscrit bien dans l'une de ces tentatives de restauration des signes du sacré à l'époque du «touttechnologique », dont Jacques Ellul et Lucien $\mathrm{Sfez}$ furent les premiers à dénoncer les effets néfastes, notamment sur le lien social. Cet ordre, en effet, cristallise non seulement des principes directifs, mais allie en outre la force spirituelle à la force physique. Car contrairement aux simples soldats, les chevaliers opèrent la synthèse des oratores et des bellatores, deux des fonctions principales qui fondent le modèle tripartite indoeuropéen décrit par Georges Dumézil. La notion de vocation qui leur est attachée leur confère une supériorité morale, fortement appréciée à l'heure où l'individu déplore un effacement progressif des idéologies transcendantes, où priment les intérêts égoïstes et les considérations positivistes. La chevalerie correspond à ce subtil mariage dont Saint-Simon reconnaissait les 
bienfaits lorsqu'il affirmait que «les moments les plus heureux pour l'espèce humaine ont été ceux où les pouvoirs spirituel et matériel se sont le mieux équilibrés ».

Au plan communicationnel, la construction de ce mythe révèle un mode de fonctionnement à rebours des tendances sociétales qui traversent les dix-neuvième et vingtième siècles. Tandis que la plupart des nations occidentales commençaient à faire de la technologie un impératif en matière de communication, le mythe chevaleresque, quant à lui, s'efforça de puiser au sein des traditions médiévales, ou encore de donner un lustre ancien à des machines modernes comme l'avion, comparé à une monture animale. Dans Critique de la communication, Lucien Sfez affirme que «pour assurer leur cohésion, les sociétés à mémoire se servent de l'histoire, les sociétés sans mémoire de la communication $\gg{ }^{15}$. Or, la franc-maçonnerie, comme l'armée de l'air, semblent bien offrir un modèle de cohésion fondé sur l'Histoire. Soucieuses de pallier les lacunes culturelles dues à leur fraîche apparition, les deux institutions fondèrent leur identité sur la mémoire collective. Face au développement exponentiel d'une "politique de la communication », qui passe "un pacte d'allégeance avec la technologie » et se trouve marquée par la répétition d'images superficielles, l'anonymat voire l'égalisation des émetteurs et des récepteurs, la vitesse de propagation des messages et l'éparpillement de signes dédoublés à l'infini, le mythe de la chevalerie propose une "politique de la mémoire » ${ }^{16}$, reposant sur un attachement aux origines et des communions symboliques. En termes médiologiques, la chevalerie repose doublement sur l'axe vertical et diachronique de la transmission des valeurs : d'abord en tant qu'ordre initiatique (ce qu'atteste l'aspect liturgique et sacramentel de l'adoubement, réalisé par un ancien de la communauté, et que tant la franc-maçonnerie ${ }^{17}$ que l'armée de l'air $^{18}$ ont repris à travers divers systèmes initiatiques et de parrainage); ensuite en tant que mythe. Ce qui n'a pas empêché des tentatives de récupération de la part du pouvoir politique, qui en fit à certaines occasions le substrat même des messages de sa communication horizontale et synchronique (comme ce fut le cas avec Napoléon Bonaparte ou Vichy). 
$\mathrm{N} \cdot \mathrm{O} \cdot \mathrm{T} \cdot \mathrm{E} \cdot \mathrm{S}$

1. Elle fit de même avec la maçonnerie opérative, rattachant ses pratiques à celles des bâtisseurs de cathédrales.

2. Pierre Mollier, La Chevalerie maçonnique : francmaçonnerie, imaginaire chevaleresque et légende templière au siècle des Lumières, Paris, Dervy, 2005. 3. Alexis Suchet, Napoléon et le management, Paris, Tallandier, 2004.

4. Jean Kaempfer, Poétique du récit de guerre, Paris, José Corti, 1998.

5. Pierre Dournel, «L'image de l'aviateur français en 1914-1918, une étude du milieu des aviateurs d'après la revue «La guerre aérienne illustrée»», Revue historique des armées, $n^{\circ}$ 4, 1975, p.73.

6. Sur cette problématique de la technologie et de ses effets en termes de délitement social, cf. Jacques Ellul (La technique ou l'enjeu du siècle. Paris, Armand Colin, 1954. Le Système technicien, Paris, Calmann-Lévy 1977) et Lucien Sfez (Critique de la communication, Paris, Seuil, 1992).

7. «Le destin médiatique des chevaliers du ciel, de Georges Guynemer à Tanguy et Laverdure », conférence donnée à Lleida, 14, 15 et 16 septembre 2000.

8. Pascal Vennesson, Les Chevaliers de l'air. Aviation et conflits au XXe siècle, Paris, Presses de la fondation nationale des sciences politiques, 1997, pp. 60-61. Pascal Vennesson remarque ainsi qu'entre 1914 et 1916, les pilotes brevetés proviennent majoritairement de la cavalerie. Ils sont presque deux fois plus nombreux que ceux qui sont issus des autres armes.

9. François Pernot, «L'Image de l'aviateur dans la presse française de 1940 à 1944 : la recherche d'un modèle », Revue historique des armées, $\mathrm{n}^{\circ} 2,1993$, pp. 85-95.
10. Cité dans Guynemer, un mythe, une histoire, Service Historique de l'Armée de l'Air, 1997 (p. 59). 11. Franco Cardini, La Culture de la guerre, Paris, Gallimard, 1992, p. 15.

12. Gilbert Durand, L'Imagination symbolique, Paris, PUF, Quadrige, 1964, p.52.

13. Jean Flori, Les Chevaliers et la Chevalerie au Moyen Âge, Paris, Hachette Littérature, 1998.

14. Paul Ricoeur, Le Conflit des interprétations. Essais d'herméneutique, Paris, éditions du Seuil, 1969 (chapitre «Herméneutique des symboles et réflexion philosophique », p. 284).

15. Lucien Sfez, Critique de la communication, Seuil,

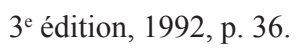

16. Lucien Sfez, La Politique symbolique, Paris, PUF, Quadrige, 1993, p. 417.

17. Lors de l'initiation, par exemple, «l'Epée flamboyante » du Vénérable Maître, posée sur la tête et les épaules de l'adepte, transmet les valeurs de la maçonnerie à l'impétrant.

18. La Présentation au Drapeau, le Baptême des Promotions, la Veillée des morts et la Cérémonie de remise des poignards, notamment. 
$R \cdot E ́ \cdot S \cdot U \cdot M \cdot E ́$

La franc-maçonnerie a hérité du myhe de la chevalerie médiévale et de ses valeurs de loyauté et de solidarité, tout comme l'aviation à ses débuts. De nos jours, une «politique de la mémoire » montre l'introduction du sacré à lépoque du tout-technologique. 
